\title{
Educação permanente como estratégia para auxiliar o profissional de saúde no acolhimento às nutrizes em sofrimento mental do banco de leite humano
}

Continuing education as a strategy to assist the health professional in welcoming the suffering mothers of the human milk bank

La educación continua como estrategia para ayudar al profesional de la salud a dar la bienvenida a las madres que sufren del banco de leche humana

Bianca Maria Innocencio Dantas da Silveira Lobo', Elaine Antunes Cortez ${ }^{2}$

Como citar esse artigo. Lobo, BMIDS; Cortez, EA. Educação permanente como estratégia para auxiliar o profissional de saúde no acolhimento às nutrizes em sofrimento mental do banco de leite humano. Revista Pró-UniverSUS. 2019 Jul./Dez.; 10 (2): 123-127.

\section{Resumo}

Apresenta-se um projeto de Pesquisa do Mestrado Profissional em Ensino da Saúde da Universidade Federal Fluminense (UFF). Objetivo geral: propor a Educação Permanente em Saúde no Banco de Leite Humano (BLH) como estratégia para contribuir para o acolhimento às nutrizes em sofrimento emocional durante o aleitamento materno. Método: trata-se de um estudo com abordagem mista (quantitativa e qualitativa) descritivo e exploratório com levantamento de dados, com abordagem metodológica qualitativa, pesquisa de campo, participativa, do tipo pesquisa-ação. Que será realizado em um BLH de um hospital Universitário do Município de Niterói (RJ). O referencial político será a Política de Educação Permanente e como referencial teórico serão utilizados os preceitos de cuidado trazidos pelo Professor Dr. Emerson Merhy, autor que dá subsídios para a temática proposta. Pretende-se para coleta de dados a utilização do questionário validado que será aplicado as Nutrizes "Edinburgh PostnatalDepressionScale (EPDS)", em um segundo momento com os profissionais de saúde serão realizadas oficinas de acordo com a metodologia da Problematização do Arco de Maguerez.

Palavras-chave: Acolhimento, Profissionais de Saúde, Bancos de Leite, Serviços de Saúde Humanização da Assistência, Sofrimento Mental.

\begin{abstract}
A research project of the Professional Master's Degree in Health Education at the Fluminense Federal University (UFF) is presented. General objective: to propose Permanent Health Education in the Human Milk Bank (BLH) as a strategy to contribute to the reception of nursing mothers in emotional distress during breastfeeding. Method: This is a descriptive and exploratory mixed approach study (quantitative and qualitative) with data collection, with qualitative methodological approach, field research, participatory, action research type. That will be held in a BLH of a University hospital in the city of Niterói (RJ). The political reference will be the Permanent Education Policy and as theoretical reference will be used the precepts of care brought by Professor Dr. Emerson Merhy, author who gives subsidies for the proposed theme. It is intended for data collection the use of validated questionnaire that will be applied to the "Edinburgh Postnatal Depression Scale (EPDS)" Nurses, in a second moment with health professionals workshops will be held according to the methodology of the Maguerez Arc Problematization.
\end{abstract}

Keywords:Welcoming; Health Professionals; Milk Banks; Health Services Humanization of Assistance;Mental Suffering.

\section{Resumen}

Se presenta un proyecto de investigación del Máster Profesional en Educación para la Salud de la Universidad Federal Fluminense (UFF). Objetivo general: proponer la Educación Permanente en Salud en el Banco de Leche Humana (BLH) como una estrategia para contribuir a la recepción de madres lactantes en angustia emocional durante la lactancia. Método: Este es un estudio descriptivo y exploratorio de enfoque mixto (cuantitativo y cualitativo) con recolección de datos, con enfoque metodológico cualitativo, investigación de campo, participativo, tipo investigación de acción. Eso se llevará a cabo en un BLH de un hospital universitario en la ciudad de Niterói (RJ). La referencia política será la Política de Educación Permanente y, como referencia teórica, se utilizarán los preceptos de atención presentados por el Profesor Dr. Emerson Merhy, autor que otorga subsidios para el tema propuesto. Está destinado a la recopilación de datos, el uso de un cuestionario validado que se aplicará a las enfermeras de la "Escala de depresión postnatal de Edimburgo (EPDS)", en un segundo momento con profesionales de la salud, se realizarán talleres de acuerdo con la metodología de la problematización del arco de Maguerez.

Palabras clave: Bienvenida, Profesionales de la salud, bancos de leche,Servicios de salud Humanización de la assistência, Sufrimiento mental. 


\section{Introdução}

A problemática que motivou o interesse da autora em realizar esta pesquisa envolve as inquietações no que tange ao acolhimento à nutriz pelo profissional de saúde. A observação da autora no atendimento clínico, como nutricionista em consultório particular e ambulatório de empresas, que a mesma teve a oportunidade de trabalhar, das questões trazidas pelas nutrizes com relação à grande dedicação que o Aleitamento Materno (AM) e o Aleitamento Materno Exclusivo (AME) representam, suas expectativas em não falhar como mãe, com cobranças externas, dificuldades pessoais e inseguranças no período pós-parto e a adaptação nem sempre descomplicada da prática do AM.

A pesquisadora por meio de sua experiência clínica observa que muitas mulheres trazem para consulta uma autopercepção de um estado mais ansioso, ou seja, um certo sofrimento mental durante esse período e relatam com frequência a falta de apoio emocional, empatia e acolhimento por parte dos Profissionais de Saúde (PS) que as assistem, tanto na rede pública, quanto na rede particular.

Não faltam referências para os nutricionistas, bem como demais profissionais da área da saúde para a defesa da prática do AME e do AM como estratégia para a manutenção da saúde física e psicoafetiva da criança. Mas entende-se ser de demasiada importância que o PS volte também seu olhar de forma cuidadosa para a mulher que amamenta, não só para garantir os benefícios inegáveis para o metabolismo e saúde da mãe, mas, sobretudo, para identificar e apoiar emocionalmente a nutriz. Principalmente, a mulher que aponta algum sofrimento emocional por ocasião da amamentação, como ansiedade, insegurança, tristeza, medo e Depressão Pós-Parto (DPP).

Apesar das conhecidas vantagens do AM e das políticas públicas desenvolvidas para evitar o desmame precoce, o Brasil ainda está aquém no cumprimento dessa recomendação. Nos últimos anos houve um aumento na prevalência da amamentação, porém, o término precoce do AM ainda pode ser considerado um importante problema de saúde pública. ${ }^{1}$

São inúmeros os esforços em apoiar e viabilizar o AM no Brasil. O Departamento de AM da Sociedade Brasileira de Pediatria (SBP), por exemplo, apoia o programa Iniciativa Hospital Amigo da Criança (IHAC) como uma maneira eficiente de incentivar e promover a amamentação. Ainda, nesse contexto o Ministério da Saúde (MS) ${ }^{2}$, instituiu, no âmbito do SUS - a portaria $\mathrm{N}^{\circ}$ 1.459 de 24 de junho de 2011 a Rede Cegonha visando implementar uma rede de cuidados para assegurar às mulheres o direito ao planejamento reprodutivo e à atenção humanizada, à gravidez, ao parto e ao puerpério, bem como assegurar às crianças o direito ao nascimento seguro e ao crescimento e desenvolvimentos saudáveis. Esta estratégia tem como finalidade estruturar e organizar a atenção à saúde materno-infantil no país e vem sendo implantada gradativamente, em todo o território nacional, iniciando sua implantação respeitando o critério epidemiológico, taxa de mortalidade infantil e razão mortalidade materna e densidade populacional.

Entretanto, é importante ressaltar que a política de apoio ao AM vai além do cuidado do binômio mãe e filho e sofre a influência de todos os atores que estão próximos a esta nova dupla. $\mathrm{O}$ ato de amamentar, manter a lactação em situações as quais os filhos não podem ser amamentados e cuidar dos transtornos, resultados da falta de manejo clínico para a amamentação, demandam cuidados e conhecimento na tecnologia específica de AM por parte dos profissionais, para então poder juntos, mãe e profissional de saúde, resolver as questões apresentadas ao longo desse processo. ${ }^{3}$

Assim, mães que apresentem transtornos de ansiedade e DPP têm menos confiança quanto à sua capacidade de amamentar e por isso estariam menos dispostas a continuar a amamentação, quando comparadas com aquelas sem sintomatologia depressiva, o estudo ainda aponta uma possível relação entre DPP e o desmame precoce. ${ }^{4}$

Portanto, é essencial que a equipe de saúde tenha o papel de acolhimento de mães e bebês, estando disponível para a escuta e para o esclarecimento de dúvidas e aflições, incentivando a troca de experiências e fazendo sempre que necessário, uma avaliação singular de cada caso. Faz-se necessário discutir as demandas da assistência em amamentação, e, se há ações práticas por tais atores, além de verificar se estão capacitados para solucionar tal demanda.

A amamentação não é um ato instintivo, por isso não deve ser creditado como conhecimentos naturais das mães para o seu sucesso, é uma prática que exige a presença de profissionais capacitados para ser ensinada. ${ }^{5}$ Os autores ressaltam que o apoio ao AM abrange também o cuidado emocional da mãe, ponderando junto a esta, maneiras de lidar com os enfrentamentos do cotidiano, ouvindo e envolvendo os seus familiares.

Com o objetivo de promover, apoiar e mobilizar o AM em 1990 a Organização Mundial da Saúde (OMS) e a United Nations Children's Fundation (UNICEF) idealizaram a IHAC visando auxiliar os funcionários dos estabelecimentos de saúde para que mudem condutas e rotinas responsáveis pelos elevados índices de desmame precoce. Para isso, foram estabelecidos os "Dez Passos para o Sucesso do AM". Todos os estabelecimentos que oferecem serviços obstétricos e cuidados aos recémnascidos, devem cumprir os dez passos. No contexto desta pesquisa, é importante destacar o décimo passo proposto: "Encorajar o estabelecimento de grupos de apoio à amamentação, para onde as mães devem ser encaminhadas por ocasião da alta hospitalar". 
Assim a relevância deste trabalho nasce de uma necessidade real em se promover apoio às nutrizes, buscando elucidar se os PS, estão de fato preparados $\mathrm{e}$ atentos à necessidade de acolher emocionalmente a mulher que amamenta e apresenta algum sofrimento emocional. Pressupõe-se que a EPS possa contribuir para a formação dos profissionais no BLH levando-os a repensar sua prática e auxiliando-os na construção de uma equipe de trabalho mais indulgente e humana. Supõemse que tal reflexão poderá despertar um movimento de autoconhecimento e de crescimento individual. ${ }^{6}$

Objetivo: propor a EPS no BLH visando contribuir para o acolhimento às nutrizes em sofrimento emocional durante o AM.

\section{Metodologia}

Trata-se de um estudo com abordagem mista (quantitativa e qualitativa) descritivo e exploratório com levantamento de dados, com abordagem metodológica qualitativa, pesquisa de campo, participativa, do tipo pesquisa-ação. $\mathrm{O}$ conjunto de dados de abordagem quantitativa e qualitativa não se opõem, pelo contrário, se complementam, pois o processo que envolve as abordagens se faz de forma dinâmica, excluindo qualquer dicotomia.?

A pesquisa qualitativa trabalha com o universo de significados, o que corresponde a um espaço mais profundo das relações, dos processos e dos fenômenos que não podem ser reduzidos à operacionalização de variáveis, preocupa-se com aspectos da realidade numa relação dinâmica entre o mundo real e o sujeito, centrando-se na compreensão das relações sociais. ${ }^{7}$

A abordagem da pesquisa-ação se caracteriza pela identificação do problema a partir dos sujeitos que identificam e o vivenciam, pesquisadores e grupo participante, para construção de movimentos consensuados de caráter social, educacional, técnico, capazes de responder com maior eficiência aos problemas reais por meio de ações facilitadoras que gerem impactos positivos. ${ }^{8}$

Os aspectos práticos de concepção, organização e operacionalização do trabalho de investigação apresentam momentos que não são rigorosamente sequenciais. O planejamento das fases deve ser flexível e passível de adequação às necessidades do pesquisador e dos participantes. ${ }^{8}$

O campo de pesquisa proposto será o BLH de um Hospital Universitário localizado na cidade de Niterói/ Rio de Janeiro, destinado à doação, armazenamento, cuidados e orientação no manejo, assim como ao apoio às nutrizes e obtenção do leite materno.

Dois grupos constituirão a população a ser pesquisada:

a) Os PS: enfermeiros, técnicos de Enfermagem, nutricionistas, médicos, que integram a equipe multidisciplinar do BLH de um Hospital Universitário.

b) As nutrizes que são assistidas no BLH de um Hospital Universitário durante o período de AM.

Todos que forem convidados a participar voluntariamente da pesquisa terão que assinar um Termo de Consentimento Livre e Esclarecido (TCLE), confirmando o interesse de participar do estudo em questão.

O desenvolvimento da pesquisa seguirá a orientação da Resolução CNS/MS, n 466/20129 que trata da ética na pesquisa em seres humanos. Para tanto, será obtida a aprovação pelo Comitê de Ética da Faculdade de Medicina da Universidade Federal Fluminense (UFF), por meio da plataforma Brasil. Após a concordância dos gestores do lócusda pesquisa e da assinatura do TCLE por parte das participantes, os instrumentos serão aplicados.

A coleta de dados acontece em etapas distintas informadas a seguir:

Etapa 1 - Observação do processo de acolhimento das nutrizes do BLH.

Etapa 2 - Utilização de um questionário para autoavaliação de DPP EPDS que se trata de um questionário de autoavaliação desenvolvido na GrãBretanha para pesquisa da DPP, traduzido e validado em diversos países, inclusive no Brasil. Esta ferramenta tem demonstrado elevada sensibilidade para a identificação do risco de DPP e transtornos emocionais, na maioria das investigações. O questionário de autoavaliação contém dez perguntas com quatro opções que são pontuadas de 0 a 3 , de acordo com a presença ou intensidade dos sintomas: humor deprimido ou disfórico, distúrbio do sono, perda do prazer, diminuição do desempenho, culpa e ideias de morte e suicídio. Como metodologia a ser aplicado com às nutrizes que aceitarem participar da pesquisa.

Etapa 3 - Os dados quantitativos serão analisados mediante o software Statistical Package for Social Sciences (SPSS) - versão 20, utilizando-se a estatística descritiva com frequência e porcentagem. Presumese que os dados qualitativos serão necessários para se justificar os resultados estatísticos do estudo e serão organizados. As nutrizes serão identificadas como $\mathrm{N}(\mathrm{N} 1, \mathrm{~N} 2, \mathrm{~N} 3, \mathrm{~N} 4, \mathrm{~N} 5, \mathrm{~N} 6, \mathrm{~N} 7 \ldots)$ e a equipe de PS serão identificadas como E1, E2, M1 e M2, P1, P2, em relação à Enfermeiras, Médicas, Psicólogos e demais profissionais respectivamente.

Etapa 4 -Serão realizadasoficinas com os PS. Nesta etapa do trabalho haverá a apresentação dos dados e resultados estatísticos coletados por meio dos questionários aplicados às nutrizes. Com o objetivo principal de apresentar dados que auxilie o PS a repensar sua prática com relação ao acolhimento das nutrizes em sofrimento emocional no BLH e traçar caminhos para uma relação de maior confiança e satisfação mútua. 
Presume-se que as oficinas terão um importante papel de escuta entre o grupo dos PS.

O escutar é "obviamente algo que vai além da capacidade auditiva de cada um. Escutar significa a disponibilidade permanente por parte do sujeito que escuta para a abertura à fala do outro, ao gesto do outro", esse autor complementa ainda que não é falando aos outros, de cima para baixo, como se quem fala tivesse toda a verdade a ser transmitida aos demais que se aprende a escutar, mas é escutando que se aprende a falar com os outros. Dessa forma, "somente quem escuta paciente e criticamente o outro, fala com ele". ${ }^{10}$

Aimportância da escuta nas relações interpessoais, pois a mesma propicia uma maior aproximação desses sujeitos que se relacionam, proporciona ainda "o reconhecimento do outro, a aceitação, a confiança mútua entre quem fala e quem escuta". ${ }^{11}$ Dessa forma, a escuta se configura em uma das pontes de aproximação do PS do BLH e a nutriz. Estabelecendo a confiança para as relações interpessoais entre quem fala para ser escutado e quem se permite escutar.

Presume-se, neste estudo, que as oficinas serão baseadas no Arco de Charles Maguerez e serão realizadas em cinco etapas:

A primeira etapa será de apresentação dos dados coletados com as nutrizes por meio do questionário para autoavaliação de DPP, EPDS para a apreciação crítica dos PS (sujeitos do grupo) e apresentação de uma situação-problema que envolva o acolhimento da nutriz em sofrimento emocional.

$\mathrm{Na}$ segunda etapa o grupo será subdividido em dois, o que objetivará a elaboração de mapas representativos da dinâmica atual de acolhimento do BLH diante da situação hipotética trazida, buscando identificar, assim, os pontos-chave apresentados por cada grupo.

Na terceira etapa quando os dois subgrupos serão convocados a refletirem juntos sobre o acolhimento que hoje acontece no cenário mediante a construção de um único mapa contendo todos os elementos identificados como interferentes e essenciais para o adequado acolhimento das nutrizes em sofrimento emocional (problematização).

Na quarta etapa, será a proposição das hipóteses, ou seja, elaboração de possíveis soluções, para as questões identificadas durante a problematização e na quinta e última etapa, será a aplicação à realidade do BLH e dos PS.

As informações serão filmadas, gravadas e transcritas de acordo com o consentimento dos participantes. Buscar-se-á ainda analisar o impacto do método das oficinas baseado no Arco de Charles Maguerez nas condutas, as principais dificuldades enfrentadas por esses profissionais e sugestões para aperfeiçoar o processo a partir da implementação do método.

\section{Resultados Esperados}

A ideia inicial é ter um produto é dar origem a um vídeo/documentário, que hoje se encontra com um título provisório: "Um colo para a mãe" que deverá ser desenvolvido e editado após a filmagem da movimentação das nutrizes no banco de leite e das oficinas com os PS, para ser utilizado no BLH em questão e em outras instituições do SUS como material de EPS em forma de tecnologia educacional, será disponibilizado o acesso pelo Youtube.

O documentário pode ser entendido como uma estratégia para revelar e dar visibilidade ao desenvolvimento da EPS do BLH, uma vez que as falas e o não dito (olhares, silêncios) das usuárias e dos PS estarão "capturados" e, assim, poderão sensibilizar outras equipes que lidam com questões similares, e, acima de tudo, estimular a reflexão e revelar caminhos de maior satisfação para ambos (nutrizes e Profissionais).

O documentário ficará disponível on-line no Youtube para uso pelas equipes de diversos bancos de leite do SUS em diferentes municípios do Brasil. Espera-se que o produto seja validado por meio de cinedebates. Onde se poderá reunir os profissionais do setor e promover a apresentação do documentário seguido de debate colhendo assim as impressões, falas e registros deste momento.

\section{Referências}

1. Almeida JM, Luz SAB, Ued FV. Apoio ao aleitamento materno pelos profissionais de saúde: revisão integrativa da literatura. Rev Paul Pediatr [Internet]. 2015 Jul/Sep [cited 2018 Aug 15]; 33(3):355-62, Available from:http://www.scielo.br/pdf/rpp/v33n3/0103-0582-rpp-33-03-0355.pdf

2. Ministério da Saúde (BR), Gabinete do Ministro. Portaria ${ }^{\circ} 1.459$ de 24 de junho de 2011. Institui no âmbito do Sistema Único de Saúde - SUS - Rede Cegonha[Internet]. Brasília: Ministério da Saúde, 2011 [cited 2018 Sep 15].Availablefrom: http://bvsms.saude.gov.br/bvs/saudelegis/gm/2011/ prt1459_24_06_2011.html

3. Branco MBLR, Alves VH, Rodrigues DP, Souza RMP, Lopes FO, Marinho TF. Proteção e apoio ao aleitamento materno: uma contribuição do banco de leite humano. RevPesquiCuid Fundam Online [Internet]. 2016 Apr/Jun [cited 2019 Apr 15]; 8(2):4300-12. Availablefrom: http://www.seer. unirio.br/index.php/cuidadofundamental/article/view/4357

4. Figueiredo B, Dias CC, Brandão S, Canário C, Nunes-Costa R. Amamentação e depressão pós-parto: revisão do estado de arte. J Pediatr [Internet]. 2013 Aug [cited 2019 May 12]; 89(4):332-8. Available from: http://www.scielo.br/pdf/jped/v89n4/v89n4a03.pdf

5. Souza SNDH, Mello DI, Ayres JRCM. O aleitamento materno na perspectiva da vulnerabilidade programática e do cuidado. Cad SaúdePública [Internet]. 2013 Jun [cited 2019 Jan 18]; 29(6):1186-94. Available from: http://www.scielo.br/pdf/csp/v29n6/a15v29n6.pdf

6. Freire P. Pedagogia do oprimido. 54. ed. Rio de Janeiro: Paz e Terra; 2013.

7. Minayo MCS, organizadora. Pesquisa social: teoria, método e criatividade. 32nd ed. Petrópolis: Vozes; 2011.

8. Thiollent M. Metodologia da pesquisa-ação. 18th. ed. São Paulo: Cortez; 2011. 
9. Ministério da Saúde (BR), Conselho Nacional de Saúde. Resolução no 466 de 12 de dezembro de 2012 [Internet]. Brasília: Ministério da Saúde; 2012 [cited 2019 Mar 21].Availablefrom:http://bvsms.saude.gov.br/bvs/ saudelegis/cns/2013/res0466_12_12_2012.html

10. Freire P. Pedagogia da autonomia: saberes necessários à prática educativa. 48th ed. São Paulo: Paz e Terra; 2014.

11. Cerqueira TCS, organizadora. (Con)Texto em escuta sensível. Brasília: Thesaurus, 2011 